\title{
Optimization Method for Extracting Stabilizer Geometry and Properties of REBCO Tapes
}

\author{
N. Riva, F. Grilli, F. Sirois, C. Lacroix, A.Akbar and B. Dutoit
}

\begin{abstract}
A good knowledge of material properties is a critical aspect for modeling high-temperature superconductor (HTS) devices. However, the electrical resistivity of coated conductors above the critical current is limited. The major challenge in characterizing this regime lies in the fact that for $I>I_{\mathrm{c}}$, heating effects and thermal instabilities can quickly destroy the conductor if nothing is done to protect it. In our previous works we proposed the overcritical current model, obtained by combining fast pulsed current measurements with finite element analysis (Uniform Current (UC) model). In this work, we assessed the impact of the uncertainties of the input parameters on the quantities calculated with the UC model (temperature and current in each layer of the tape). Firstly, sensitivity and uncertainty analyses were performed and it was found that the input parameters that mostly affect the UC model are the electrical resistivity and the thickness of the silver layer. Afterwards, an optimization method to correctly estimate the geometry and the resistivity of the silver layer was developed. This method combined experimental measurements of resistance $R(T)$ of the tape and pulsed current measurement. The development of this strategy allowed us to determine the parameters that significantly impact the UC model and helps to minimize their uncertainties. This can enable a precise estimation of the overcritical current resistivity. $10 \%$
\end{abstract}

Index Terms-HTS, REBCO, optimization, sensitivity, uncertainty overcritical current, pulsed current measurements, finite element analysis

\section{INTRODUCTION}

$\mathbf{H}$ IGH-Temperature Superconductor (HTS), especially REBCO commercial tapes, are an emerging technology holding immense potential for future applications such as in the fields of medicine and energy. A good knowledge of their behavior in the overcritical current regime is necessary to develop quality simulation tools. In previous years, the numerical models used to describe REBCO commercial tapes' electrical resistivity for devices working near and above the critical current $\left(I>I_{\mathrm{c}}\right)$, have been shown to be inaccurate or very empirical [1]-[3]. The quick destruction of the conductor due to heating effects and thermal instabilities when $I>I_{\mathrm{c}}$ is the major obstacle for characterizing the overcritical current regime. Moreover, due to the current sharing between the layers, it is difficult to know the amount of current carried by the superconducting layer and its resistivity. In our previous works [4], [5], we combined pulsed current measurements

This project has received funding from the Swiss Federal Office of Energy SFOE under grant agreement SI/500193-02.

N. Riva, A.Akbar and B. Dutoit are with Ecole Polytechnique Fédérale de Lausanne, Switzerland (e-mail:nicolo.riva@epfl.ch, bertrand.dutoit@epfl.ch)

F. Sirois and C. Lacroix are with Polytechnique Montréal, Canada (f.sirois@polymtl.ca, c.lacroix@polymtl.ca)

F. Grilli is with Karlsruhe Institute of Technology (francesco.grilli@kit.edu)
(PCM) with a 2-D thermal model (which we named Uniform Current (UC) model), in order to estimate the temperature over time for each layer of the REBCO tape, as well as the amount of current flowing in each layer. We demonstrated that it was possible to overcome the challenges and to extract the overcritical current resistivity curves of REBCO tapes. A critical aspect of the UC model is the influence of the geometry and the uncertainty in material properties, on the simulated quantities of interest (temperature, current and resistivity). In this work, we present a post-processing method capable of investigating these aspects.

In Section II, we present sensitivity and uncertainty analyses to obtain an analytic estimation of the sensitivity of the quantities of interest (temperature and current sharing), and the variation of such quantities due to the variability of the input parameters (material and geometric properties). Specifically, the sensitivity analysis allows quantifying the variation in the quantities of interest due to the variability of the sensitive parameters. The knowledge of the sample's exact geometry and its resistivity is therefore essential for the UC model. However, such measurements can be complicated and timeconsuming. In Section III we present an optimization method developed to avoid excessive computational efforts. Experimental measurements of resistance $R$ vs temperature $T$ of the tape and pulsed current measurements were accompanied by the use of an algorithm developed to correctly estimate the geometry and the resistivity of the silver layer. This method was applied on several samples. In this paper we present the selected case for the SuperPower tape SF4050 (4 mm wide tape, with a $I_{\mathrm{c}}=110 \mathrm{~A}$ at $77 \mathrm{~K}$ and self-field conditions).

\section{Sensitivity And UnCertainty Analysis}

In this Section, we present the sensitivity and uncertainty analyses performed on the current sharing and the resistivity calculated with the UC model. ${ }^{1}$

\section{A. Sensitivity Analysis}

The resistivity calculated according to the UC model [4], [5] can be written as

$$
\rho_{\mathrm{UC}}=\frac{V_{\mathrm{meas}}}{I_{\mathrm{REBCO}}} \cdot \frac{S_{\mathrm{REBCO}}}{L},
$$

\footnotetext{
${ }^{1}$ As regards the temperature, there is no direct analytical formula to estimate the impact of the uncertainties on a partial derivative equation (PDE). We did not consider it here.
} 


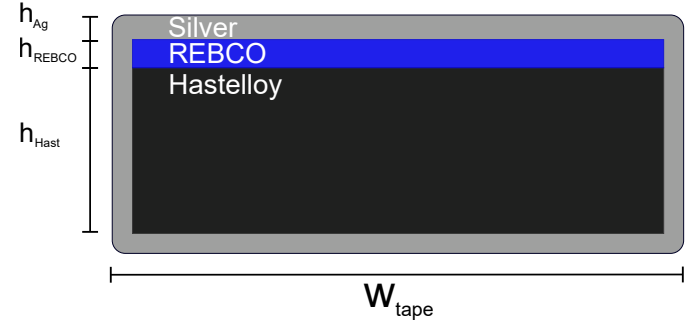

Fig. 1: Cross section of the sample with the definition of the geometrical parameters.

where $V_{\text {meas }}$ is the voltage measured over a given length $L$ and $S_{\mathrm{REBCO}}$ is the cross-section of the REBCO layer. The current in the REBCO layer $I_{\mathrm{REBCO}}$ is

$$
I_{\mathrm{REBCO}}=I_{\mathrm{tot}}-I_{\mathrm{Ag}}-I_{\text {Hast }} \text {, }
$$

where the current in each layer $k$ (Fig. 1) is

$$
I_{k}=J \cdot S_{k}=\frac{E}{\rho_{k}(T)} \cdot\left(h_{k} \cdot w_{k}\right) \text {. }
$$

Recalling that the electric field can be written as $E=$ $V_{\text {meas }} / L$, Eq. (1) can be expressed in terms of Eq. (2) and (3) as follows:

$$
\begin{aligned}
& \rho_{\mathrm{UC}}=V_{\text {meas }} \cdot \frac{h_{\mathrm{REBCO}} w_{\mathrm{REBCO}}}{L} . \\
& \frac{1}{\left[I_{\text {tot }}-\frac{V_{\text {meas }}}{L}\left(\frac{h_{\mathrm{Ag}} w_{\mathrm{Ag}}}{\rho_{\mathrm{Ag}}(T)}+\frac{h_{\text {Hast }} w_{\text {Hast }}}{\rho_{\text {Hast }}(T)}\right)\right]} .
\end{aligned}
$$

Eq. (4) allows identifying the parameters whose uncertainties affects the calculated resitivity. ${ }^{2}$ In order to obtain an analytic estimation of the sensitivity of the results on the input parameters, in Eq. (2) and (4), we used the partial derivatives method with respect to the inputs [6]. For the thickness of the silver $h_{\mathrm{Ag}}$ we have:

$$
\begin{aligned}
& \frac{\partial I_{\mathrm{REBCO}}}{\partial h_{\mathrm{Ag}}}=-\frac{V_{\mathrm{meas}} w_{\mathrm{Ag}}}{L \rho_{\mathrm{Ag}}(T)}, \\
& \frac{\partial \rho_{\mathrm{UC}}}{\partial h_{\mathrm{Ag}}}=+V_{\text {meas }}^{2} \cdot \frac{w_{\mathrm{Ag}} h_{\mathrm{REBCO}} w_{\mathrm{REBCO}}}{\rho_{\mathrm{Ag}}(T) L^{2}} . \\
& 1 \\
& \overline{\left[I_{\text {tot }}-\frac{V_{\text {meas }}}{L}\left(\frac{h_{\mathrm{Ag}} w_{\mathrm{Ag}}}{\rho_{\mathrm{Ag}}(T)}+\frac{h_{\text {Hast }} w_{\text {Hast }}}{\rho_{\text {Hast }}(T)}\right)\right]^{2}} .
\end{aligned}
$$

From Eq. (5) and Eq. (6) we observe that with an increasing silver thickness $h_{\mathrm{Ag}}$, the estimated current in the REBCO decreases, while the estimated REBCO resistivity $\rho_{\mathrm{UC}}$ increases. With a similar analysis, it can be showed that a larger resistivity of the silver layer results in a smaller amount of current into the silver layer, and thus, a higher estimated current in the REBCO layer and a lower resistivity of the REBCO material.

In addition, the variation of the quantities of interest with respect to $h_{\mathrm{Ag}}$ also depends on the considered dynamics (i.e.,

\footnotetext{
${ }^{2}$ Note that $w_{A g}=w_{\text {Hast }}=w_{\text {tape }}$.
}

the measured voltage $V_{\text {meas }}$ and current $I_{\text {tot }}$ ). This means that, in terms of sensitivity to the silver thickness, the higher the voltage (or current) amplitude, the more sensitive the calculated current and resistivity.

The same sensitivity analysis can be repeated for other parameters (i.e., $h_{\text {Hast }}, h_{\mathrm{REBCO}}, \rho_{\mathrm{Ag}}(T), \rho_{\text {Hast }}(T), C_{\mathrm{p} \text {, Hast }}(T)$, etc...). Then, it is possible to estimate which parameters drive the majority of variation in the output calculated quantity. This allows excluding some of the parameters from the uncertainty analysis that follows. We use the notation $\alpha_{\mathrm{Q}, \mathrm{par}}$, defined as

$$
\alpha_{\mathrm{Q}, \mathrm{par}}=\frac{\partial \mathrm{Q}}{\partial \mathrm{par}},
$$

to indicate the variation of the quantity "Q" (current, resistivity) with respect to the parameter "par" $\left(h_{\mathrm{Ag}}, h_{\mathrm{Hast}}\right.$ etc...). If we consider $\alpha_{I_{\mathrm{REBCO}}, h_{\mathrm{Ag}}}$ and $\alpha_{I_{\mathrm{REBCO}}, h_{\mathrm{Hast}}}$, we can assess how much the variation in silver thickness affects the current calculated with UC model compared to the variation in Hastelloy thickness as

$$
\frac{\alpha_{I_{\mathrm{REBCO}}, h_{\mathrm{Ag}}}}{\alpha_{I_{\mathrm{REBCO}}, h_{\mathrm{Hast}}}}=\frac{w_{\mathrm{Ag}}}{\rho_{\mathrm{Ag}}} \frac{\rho_{\text {Hast }}}{w_{\text {Hast }}} \approx \frac{1.2 \cdot 10^{-6}}{2 \cdot 10^{-9}} \approx 600 .
$$

Eq. (8) means that a variation of the silver thickness layer influences the calculated current in the REBCO remarkably more than a variation of the Hastelloy thickness layer. Using the same analysis, this method we can determine that some of the input parameters have a stronger influence on the calculated output quantities with respect to others (e.g., $\left.\alpha_{I_{\mathrm{REBCO}}, \rho_{\mathrm{Ag}}} / \alpha_{I_{\mathrm{REBCO}}, \rho_{\mathrm{Hast}}}=10^{5}\right)$. As a result of this analysis, we obtained that the input parameters that mostly affect the UC model are the electrical resistivity $\rho_{\mathrm{Ag}}$ and the thickness of the silver layer $h_{\mathrm{Ag}}$.

\section{B. Uncertainty Analysis}

An uncertainty analysis was performed using the UC model, and varying the sensitive parameters (electrical resistivity and thickness of the silver layer) within a uniform distribution of $\pm 10 \%$ with respect to the initial values. The uncertainty analysis allowed quantifying the variation of the quantities of interest (resistivity, temperature, and current) due to the variation in the sensitive parameters (resistivity and thickness of the silver). In Fig. 2 (left), we present the overall measured current (continuous blue line) and the current in the REBCO layer for three values of surrounding silver stabilizer (dashed lines). In Fig. 2 (right), we present the simulated average temperature of the REBCO layer for the same three values. If the UC model is run with a surrounding silver of $1 \mu \mathrm{m}$, the estimated current in the REBCO layer drops to $\simeq 0 \mathrm{~A}$ (central yellow line) at around $65 \mu \mathrm{s}$, when the corresponding temperature is $92 \mathrm{~K}$. The UC model correctly estimates the current in the REBCO layer, when it drops to a minimum (critical temperature of REBCO tapes [7], [8]). However, a variation of $+0.1 \mu \mathrm{m}$ of surrounding silver stabilizer $(1.1 \mu \mathrm{m}$ overall) leads to a large error on the current calculated in the REBCO layer, i.e., the current in the REBCO layer drops to $-38 \mathrm{~A}$ (dashed green line), which has no physical meaning. 

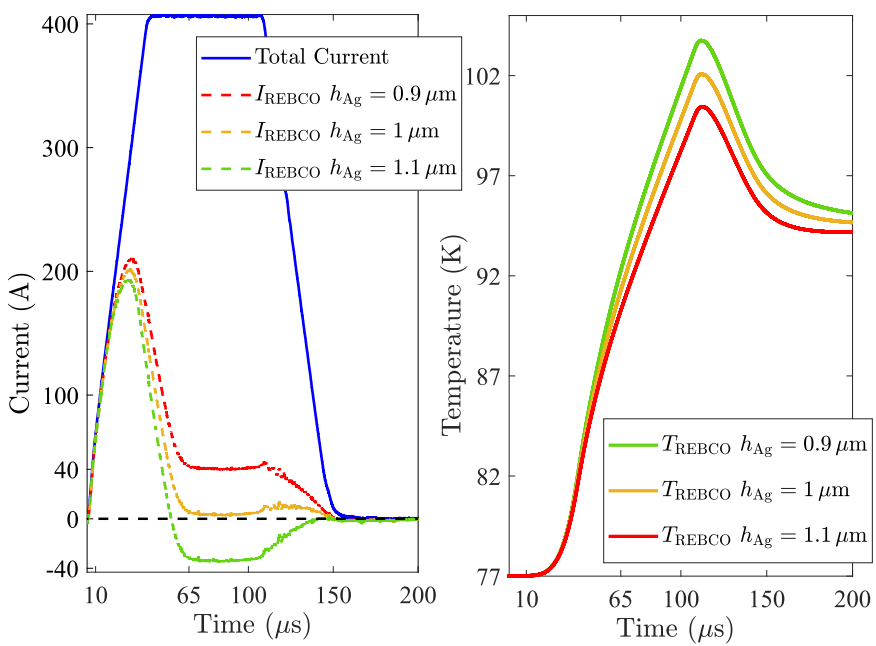

Fig. 2: Sensitivity simulation of the UC model on a pulse performed on the SuperPower sample. .

This analysis demonstrates that a good knowledge of the material properties is crucial. However, the exact geometry and resistivity of the sample are not precisely known, due to the sample purity (residual-resistivity ration RRR) and the difficulty of measuring the actual silver thickness. In the next Section, we present a method to address this particular problem.

\section{Optimization Method: Results and Discussion}

In this approach, experimental measurements of $R(T)$ of the tape and quenched voltage PCM (all the current flows into the silver and Hastelloy matrix) are coupled to an algorithm aimed at estimating the geometry and the resistivity of the silver layer. The first step requires a good initial guess of the involved quantities (namely $h_{\mathrm{Ag}}$ and $\rho_{\mathrm{Ag}}(T)$ ). This is done by combining the characteristics provided by the manufacturer on the data-sheet with direct measurements of $R$ vs $T$ (carried out with an experimental setup similar to that described in [9]). The injected current to measure $R$ vs $T$ is very small $(100 \mathrm{~mA})$, and we can assume that the acquired voltage signal is given by the silver layer only. Hence, it is possible to describe the electrical resistance of the tape as that of the silver with a temperature dependent linear fit:

$$
R_{\mathrm{Ag}}(T)=R_{\mathrm{Ag}, 0}+\beta_{\mathrm{Ag}} \cdot\left(T-T_{\mathrm{c}}\right) .
$$

In Fig. 3(left), we report the measured resistance vs temperature. The electrical resistance of the tape embeds the information about both silver thickness and resistivity:

$$
R_{\mathrm{Ag}}(T)=\left(\widetilde{\rho}_{\mathrm{Ag}, 0}+\widetilde{\alpha}_{\mathrm{Ag}} \cdot\left(T-T_{\mathrm{c}}\right)\right) \cdot \frac{l_{\text {meas }}}{\widetilde{h}_{\mathrm{tot}, \mathrm{Ag}} \cdot w_{\text {tape }}},
$$

with $l_{\text {meas }}=10 \mathrm{~cm}$ being the length of the measured section for the $R$ vs $T$ measurements, and $w_{\text {tape }}=4 \mathrm{~mm}$ the width of the tape. The notation $\sim$ (tilde) indicates the unknown parameters that we investigate with the optimization method. For the analyzed sample, the amount of silver declared on the data-sheet was of $h_{\mathrm{tot}, \mathrm{Ag}}=2.2 \mu \mathrm{m}(1.1 \mu \mathrm{m}$ surrounding silver). The parameters in Eq. (10) can be estimated by equating the fit calculated in Fig. 3(left) (i.e. Eq. (9)), and the datasheet:

$$
\begin{aligned}
\widetilde{\rho}_{\mathrm{Ag}, 0} & =\frac{R_{\mathrm{Ag}, 0} \cdot \widetilde{h}_{\mathrm{tot}, \mathrm{Ag}} \cdot w_{\text {tape }}}{l_{\text {meas }}}=0.3943 \mu \Omega \mathrm{cm} \\
\widetilde{\alpha}_{\mathrm{Ag}} & =\frac{\beta_{\mathrm{Ag}} \cdot \widetilde{h}_{\mathrm{tot}, \mathrm{Ag}} \cdot w_{\text {tape }}}{l_{\text {meas }}}=0.0049 \mu \Omega \mathrm{cm} \mathrm{K}^{-1} .
\end{aligned}
$$

With these values, the UC model predicts that the current in the REBCO drops below zero, as shown in Fig. 4 (black line). This means that the set of used parameters overestimates the actual amount of silver (or underestimates its resistivity). The overestimation of silver thickness leads to an excessive value of current calculated for the silver layer, and thus a negative current in the REBCO layer. The initial guess need to be adjusted and optimized for a better result.

The second step of the optimization method involves the analysis of long PCM $(t>100 \mu \mathrm{s})$ at large current amplitudes $\left(I_{\text {tot }}>3 I_{\mathrm{c}}\right)$, for which the sample quenches and the current flows entirely in the stabilizer matrix. In Fig. 3(right), we report the specific case of one pulse performed on the sample. When the pulsed current amplitude is as high as $400 \mathrm{~A}$, we cannot neglect the contribution of the Hastelloy layer since part of the current flows in there. The overall resistivity of the sample can be represented as follows:

$$
R_{\text {tape }}(T) \simeq \frac{R_{\mathrm{Ag}}(T) \cdot R_{\text {Hast }}(T)}{R_{\mathrm{Ag}}(T)+R_{\text {Hast }}(T)},
$$

where $R_{\mathrm{Ag}}(T)$ and $R_{\text {Hast }}(T)$ are the resistance of the silver and Hastelloy, respectively. We can express $R_{\mathrm{Ag}}(T)$ and $R_{\text {Hast }}(T)$ with a relationship similar to Eq. (9).

If we assume that the Hastelloy's temperature-dependence resistivity is negligible $\left(\rho_{\text {Hast }}(T)=\right.$ const $=1.23$. $10^{-6} \Omega \mathrm{m}$ ) [10], then the electrical resistivity of the silver depends linearly on the temperature $\rho_{\mathrm{Ag}}(T)=\rho_{\mathrm{Ag}, 0}+\alpha_{\mathrm{Ag}}$. $\left(T-T_{\mathrm{c}}\right)$, so we can rearrange Eq. (12) with the analytic expression:

$$
\begin{aligned}
& R_{\text {tape }}(T)=\frac{l_{\text {tape }} \cdot \rho_{\text {Hast }}}{w_{\text {tape }}} . \\
& \frac{\widetilde{\rho}_{\text {Ag }, 0}+\widetilde{\alpha}_{\mathrm{Ag}} \cdot\left(T-T_{\mathrm{c}}\right)}{\left[\widetilde{\rho}_{\mathrm{Ag}, 0}+\widetilde{\alpha}_{\mathrm{Ag}} \cdot\left(T-T_{\mathrm{c}}\right)\right] h_{\mathrm{tot}, \text { Hast }}+\rho_{\text {Hast }} \cdot \widetilde{h}_{\mathrm{tot}, \mathrm{Ag}}},
\end{aligned}
$$

where $l_{\text {tape }}=5 \mathrm{~mm}, w_{\text {tape }}=4 \mathrm{~mm}$ are the length and width of the measured section, $h_{\text {tot,Hast }}=50 \mu \mathrm{m}$ to $90 \mu \mathrm{m}$ is the thickness of the Hastelloy layer. The parameter $T_{\mathrm{c}}$, which varies from $89 \mathrm{~K}$ to $92 \mathrm{~K}$, is the reference temperature (in this case the critical temperature of the analyzed tape). We have $\widetilde{h}_{\text {tot,Ag }}$ as the total thickness of the silver layer, i.e. if the surrounding silver layer is $1 \mu \mathrm{m}, h_{\mathrm{tot}, \mathrm{Ag}}=2 \mu \mathrm{m}$. The parameter $\widetilde{\rho}_{\mathrm{Ag}, 0}$ is the silver resistivity at the reference temperature, and $\widetilde{\alpha}_{\mathrm{Ag}}$ is the linear temperature coefficient $\left(\Omega \mathrm{m} \mathrm{K}^{-1}\right)$. With the analytic form in Eq. (13) we can formulate and solve a nonlinear least squares fitting problem, matching the quenched voltage curves with the analytic function (i.e., minimizing the residuals calculated as $\left.\left|R_{\exp }-R_{\text {tape }}\right|\right)$.

The problem is defined as follows: 

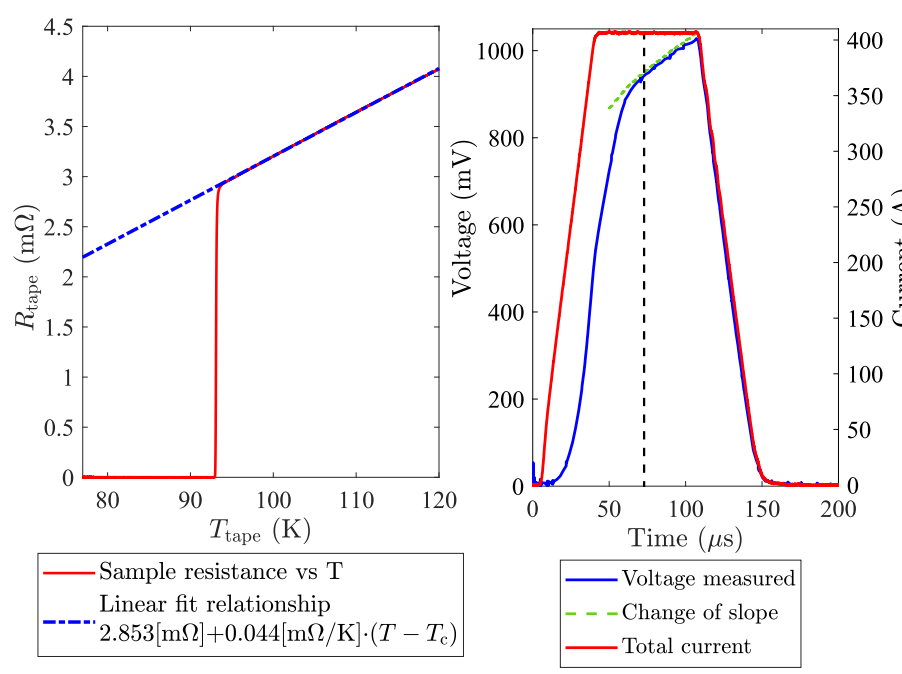

Fig. 3: Measured tape resistance vs temperature of the investigated sample (left), and example of selected pulse exhibiting a change of slope of the measured voltage (right).

1) Given a set of $n_{\text {tot }}$ experimental points $\left\{T_{\exp , \mathrm{k}}, R_{\exp , \mathrm{k}}\right\}$ ( $R$ vs $T+$ quenched PCM);

2) Given the analytic model function in Eq. (13);

3) Find $\left(\left[\widetilde{\rho}_{\mathrm{Ag}, 0}, \widetilde{\alpha}_{\mathrm{Ag}}, \widetilde{h}_{\mathrm{tot}, \mathrm{Ag}}\right]\right)$ describing $R_{\text {tape }}$ and matching the quenched PCM such that the following residuals are minimized:

$$
\left\{\sum_{n=1}^{n_{\text {tot }}}\left[R_{\text {exp }, \mathrm{k}}-R_{\text {tape }}\left(\left[\widetilde{\rho}_{\mathrm{Ag}, 0}, \widetilde{\alpha}_{\mathrm{Ag}}, \widetilde{h}_{\mathrm{tot}, \mathrm{Ag}}\right], T_{\text {exp }, \mathrm{k}}\right)\right]^{2}\right\}
$$

The algorithm formulated above is implemented in MatLab [11] and performs several iterations (non-linear least square fit) until the set of experimental points $\left\{T_{\exp , \mathrm{k}}, R_{\exp , \mathrm{k}}\right\}$ matches the analytic function in Eq. (13). When fitting multiple parameters of a non-linear function, the $\chi^{2}$ surface is likely to have multiple minima [12], possibly leading to mathematically correct but nonphysical solutions. A good initial guess and setting the right upper and lower limits of the searched parameters, is of paramount importance. Taking the values from Eq. (11) as the initial guess, we assume the upper and lower bounds to vary by $\pm 20 \%$ with respect to the initial guess (e.g. $\left.\widetilde{\rho}_{\mathrm{Ag}, 0}=(2 \pm 0.4) \mu \mathrm{m}\right)$. The non-linear least squares fitting problem for this specific sample returns the optimal parameters $\widetilde{h}_{\mathrm{tot}, \mathrm{Ag}}^{\mathrm{op}}=1.92 \mu \mathrm{m}, \widetilde{\rho}_{\mathrm{Ag}, 0}^{\mathrm{op}}=0.3361 \mu \Omega \mathrm{cm}$, and, $\widetilde{\alpha}_{\mathrm{Ag}}^{\mathrm{op}}=0.0052 \mu \Omega \mathrm{cm} \mathrm{K}^{-1}$. If the UC model is rerun with such parameters, we obtain the results reported in Fig. 4 (green line). Here the difference between using the optimized parameters with respect to the non-optimized parameters can be appreciated. Specifically, while the initial parameters poorly estimate the REBCO layer's current, with the new set of parameters the current in the REBCO layer reaches $\simeq 0 \mathrm{~A}$ and remains constant afterwards. This result is a good indication that the set of parameters obtained with the algorithm of Eq. (14), offer a better approximation of the actual thickness of the silver layer.

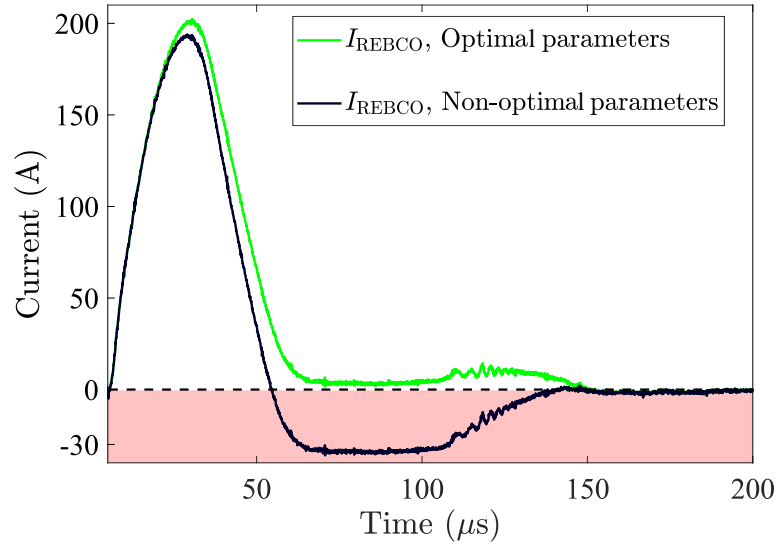

Fig. 4: Current calculated in the REBCO layer using nonoptimal (black curve) and optimal parameters (green curve). The non-optimal parameters lead to nonphysical solutions (red-shaded region).

Here, it is necessary to comment about the general validity of this method. This method was applied on a limited number of characterized samples (4 samples overall). In Tab. I, we report the optimal values obtained by applying the nonlinear LSQ fitting problem, and the nominal amount of total thickness of the silver layer provided by the manufacturer on the data-sheet. The values are consistent with each other, especially as regards of $\widetilde{\rho}_{\mathrm{Ag}, 0}$. In the case of the sample from THEVA, even if the silver thickness was much higher than the other samples, the method seems to work adequately well. However, we note that $\widetilde{\alpha}_{\mathrm{Ag}}$ is higher for that particular sample and this should be treated with caution. The reason for this discrepancy has to be further investigated. Overall, the obtained values are in good agreement with those reported in the literature [13]-[15].

TABLE I: Optimal AND Nominal Values.

\begin{tabular}{lllll}
\hline Sample & $\begin{array}{l}\tilde{\rho}_{\mathrm{Ag}, 0}^{\mathrm{op}} \\
{[\mu \Omega \mathrm{cm}]}\end{array}$ & $\begin{array}{l}\widetilde{\alpha}_{\mathrm{Ag}}^{\mathrm{op}} \\
{\left[\mu \Omega \mathrm{cm} \mathrm{K}{ }^{-1}\right]}\end{array}$ & $\begin{array}{l}\widetilde{h}_{\mathrm{tot}, \mathrm{Ag}}^{\mathrm{op}} \\
{[\mu \mathrm{m}]}\end{array}$ & $\begin{array}{l}h_{\mathrm{tot}, \mathrm{Ag}}^{\text {nom }} \\
{[\mu \mathrm{m}]}\end{array}$ \\
\hline $\begin{array}{l}\text { SuperPower } \\
(\mathrm{SF} 4050)\end{array}$ & 0.3361 & 0.0052 & 1.92 & 2 to 3 \\
\hline $\begin{array}{l}\text { SuperPower } \\
\text { (SF4050) }\end{array}$ & 0.4200 & 0.0060 & 2.2 & 2 to 3 \\
\hline $\begin{array}{l}\text { THEVA } \\
(\mathrm{TPL} 1100)\end{array}$ & 0.3676 & 0.0082 & 18 & 15 to 19 \\
\hline SuperOx & 0.3957 & 0.0048 & 1.8 & 2 to 3 \\
\hline
\end{tabular}

Finally, the entire post-processing method relies on the use of pulsed current measurements on the $\mu$ s scale. In order to confirm the reliability of the method, other voltage curves obtained with different measurement systems should be tested (e.g., ms pulses).

\section{CONCLUSION}

In this work, we presented an analysis of the impact of the UC model's input parameters (material and geometry properties) on the calculated quantities (current sharing, RE$\mathrm{BCO}$ resistivity). We found that the parameters that drive the 
majority of the variation in the outputs calculated with the UC model are the surrounding silver and its resistivity. An uncertainty analysis showed that, by a wrong estimation of the silver layer of $0.1 \mu \mathrm{m}$, the current in the REBCO layer deviates from the expected value by $40 \mathrm{~A}$. Finally, we developed a method that determines the parameters that mostly affect the UC model and helps minimize their uncertainties. This enables a more precise estimation of the overcritical current resistivity.

\section{REFERENCES}

[1] C. Im, S. An, J. Bang, J. Kim, K. R. Bhattarai, K. L. Kim, K. Kim, and S. Hahn, "An Inverse Calculation Study on Post-Quench Behavior of a No-Insulation REBCO Insert," IEEE Transactions on Applied Superconductivity, vol. 30, no. 4, pp. 1-5, jun 2020.

[2] W. T. B. de Sousa, A. Polasek, R. Dias, C. F. T. Matt, and R. de Andrade, "Thermal-electrical analogy for simulations of superconducting fault current limiters," Cryogenics, vol. 62, pp. 97109, 2014. [Online]. Available: http://www.sciencedirect.com/science/ article/pii/S0011227514000885

[3] J.-B. Song, S. Hahn, T. Lécrevisse, J. Voccio, J. Bascuñán, and Y. Iwasa, "Over-current quench test and self-protecting behavior of a 7 T/78 mm multi-width no-insulation $\{$ REBCO $\}$ magnet at $4.2 \mathrm{~K}$,' Superconductor Science and Technology, vol. 28, no. 11, p. 114001, sep 2015.

[4] S. Richard, F. Sirois, and C. Lacroix, "Post-processing method for extracting the resistivity of Rare-Earth Barium Copper Oxide (REBCO) coated conductors in over-critical current conditions from ultra-fast V-I pulsed current measurements," Journal of Applied Physics, vol. 126, no. 023902, p. 023902, 2019.

[5] N. Riva, S. Richard, F. Sirois, C. Lacroix, B. Dutoit, and F. Grilli, "Overcritical Current Resistivity of YBCO-Coated Conductors Through Combination of PCM and Finite-Element Analysis," IEEE Transactions on Applied Superconductivity, vol. 29, no. 5, p. 6601705, 2019.

[6] A. Saltelli, M. Ratto, T. Andres, F. Campolongo, J. Cariboni, D. Gatelli, M. Saisana, and S. Tarantola, Global Sensitivity Analysis. Wiley, 2008, vol. 76 , no. 3 .

[7] M. K. Wu, J. R. Ashburn, C. J. Torng, P. H. Hor, R. L. Meng, L. Gao, Z. J. Huang, Y. Q. Wang, and C. W. Chu, "Superconductivity at $93 \mathrm{~K}$ in a new mixed-phase Y-Ba-Cu-O compound system at ambient pressure," Physical Review Letters, vol. 58, no. 9, pp. 908-910, mar 1987.

[8] D. W. Hazelton, "2G HTS Wire Development at SuperPower," SuperPower, Tech. Rep., 2016.

[9] N. Riva, F. Sirois, C. Lacroix, W. T. B. de Sousa, B. Dutoit, and F. Grilli, "Resistivity of REBCO tapes in overcritical current regime: impact on superconducting fault current limiter modeling," Superconductor Science and Technology, vol. 33, no. 11, p. 114008, oct 2020.

[10] J. Lu, E. Choi, and H. Zhou, "Physical properties of Hastelloy C-276 at cryogenic temperatures," Journal of Applied Physics, vol. 103, no. 6, pp. 64906-64 908, mar 2008.

[11] MatLab, MATLAB R2019b, The Mathworks, Inc., Natick, Massachusetts, 2017.

[12] E. J. Schlossmacher, "An Iterative Technique for Absolute Deviations Curve Fitting," Journal of the American Statistical Association, vol. 68, no. 344, pp. 857-859, 1973.

[13] F. Roy, B. Dutoit, F. Grilli, and F. Sirois, "Magneto-thermal finite element modeling of 2nd generation HTS for FCL design purposes," Journal of Physics: Conference Series, vol. 97, no. 1, 2008.

[14] F. Roy, "Modeling and Characterization of Coated Conductors Applied to the Design of Modeling and Characterization of Coated Conductors Applied to the Design of Superconducting Fault Current Limiters," PhD Thesis, EPFL, 2010.

[15] S. Zou, V. M. R. Zermeño, A. Baskys, A. Patel, F. Grilli, and B. A. Glowacki, "Simulation and experiments of stacks of high temperature superconducting coated conductors magnetized by pulsed field magnetization with multi-pulse technique," Superconductor Science and Technology, vol. 30, no. 1, p. 014010, 2017. 